\title{
Representation and Similarity: Suárez on Necessary and Sufficient Conditions of Scientific Representation
}

\author{
Michael Poznic ${ }^{1}$
}

Published online: 22 July 2015

(C) The Author(s) 2015. This article is published with open access at Springerlink.com

\begin{abstract}
The notion of scientific representation plays a central role in current debates on modeling in the sciences. One or maybe the major epistemic virtue of successful models is their capacity to adequately represent specific phenomena or target systems. According to similarity views of scientific representation, models should be similar to their corresponding targets in order to represent them. In this paper, Suárez's arguments against similarity views of representation will be scrutinized. The upshot is that the intuition that scientific representation involves similarity is not refuted by the arguments. The arguments do not make the case for the strong claim that similarity between vehicles and targets is neither necessary nor sufficient for scientific representation. Especially, one claim that a similarity view wants to uphold, still, is the following thesis: only if a vehicle is similar to a target in relevant respects and to a specific degree of similarity then the vehicle is a scientific representation of that target.
\end{abstract}

Keywords Models $\cdot$ Scientific representation $\cdot$ Similarity $\cdot$ Isomorphism

\section{Introduction}

The activity of modeling primarily aims at representing certain target systems. It seems to be obvious to some philosophers and to many scientists that models have to be similar to their corresponding targets in order to represent them. Although the notion of similarity has its defenders and quiet friends there are a great number of opponents. In philosophy of science's debates on representation and modeling, Mauricio Suárez's arguments against similarity views of scientific representation are frequently mentioned. In detail, these

Michael Poznic

M.Poznic@tudelft.nl

1 Faculty of Technology, Policy and Management, Delft University of Technology, Delft, The Netherlands 
arguments are put forward in Suárez (2003). Although they are often cited, they are but rarely discussed extensively. ${ }^{1}$ Are they really knockdown arguments against any similarity account of representation in the sciences? In particular, one question raised in this paper is whether the arguments show that it is impossible to establish an account of scientific representation that comprises similarity as a necessary condition for a vehicle to represent a target. In the following, I will first give a concise survey of the background of these debates (Sect. 2). Secondly, Suárez's own position will be characterized briefly and I will discuss a distinction made by Suárez between two different kinds of relations, namely the distinction between means and constituents of representation (Sect. 3). Afterwards, I will introduce Suárez's reductionist and non-reductionist opponents and I will also discuss his arguments against similarity as a sufficient condition for representation (Sect. 4). Finally, I will analyze his arguments against similarity as a necessary condition for scientific representation (Sect. 5) and the last paragraph contains a concluding discussion (Sect. 6).

\section{Theories of Scientific Representation}

The overall aim of using models in the sciences is to gain epistemic benefits. The activity of modeling has an epistemic function and this function seems only to be fulfilled by models that are in some appropriate way connected to reality. It almost seems inevitable to ask: how can scientists gain knowledge of the natural world by studying models if these models do not share certain features with their target systems? Notions of resemblance or similarity appear relevant when explaining why scientific models can be used to foster knowledge of certain phenomena. ${ }^{2}$

In this vein, several accounts of representation have emerged in the last years. They focus on the information a representational vehicle is able to convey. These "informational" views try to explain what representation is by pointing to similarity relations between vehicles and targets, at least in one way or the other (cf. Chakravartty 2010). There are different variants of this position; some use the formal notions of isomorphism, partial isomorphism, homomorphism, ${ }^{3}$ or other mathematical mappings. Others speak of analogies, similes, or resemblances. Either way, the relation between vehicles and targets comprises structural similarities in most cases.

In seeming contrast to these informational views, other approaches focus on the activity of representing. These approaches specifically start their study of representation with the use of representational vehicles. According to these views, the intended uses of scientific models or other representational vehicles by scientists are prior to any established relation

\footnotetext{
${ }^{1}$ One exception is Bueno and French (2011) and, there, the authors deal with Suarez's arguments as challenges to their own formal account of representation. In Contessa (2007), it is argued that Suárez's arguments should be interpreted as only opposing conceptions of faithful epistemic representation in contrast to conceptions of epistemic representation. I assume that the notion of scientific representation that I am elaborating on is neither equivalent to epistemic representation nor to faithful epistemic representation in Contessa's sense.

2 I use "similarity" and "resemblance" as synonymous and I will follow Suárez's characterization of similarity. A vehicle and a target are similar if and only if they share some of their properties (cf. Suárez 2003, 227).

3 There is a debate on whether homomorphisms are instances of similarity. Because similarity is reflexive and symmetric and homomorphism is not, as Bartels (2006) argues, his homomorphism account of representation evades problems that a similarity account faces. Chakravartty (2010), on the other hand, subsumes homomorphism accounts to the informational camp and claims that homomorphism can be described in terms of structural similarity.
} 
of representation. These views are labeled differently. "Pragmatic," "deflationary" or "functional" are all adjectives that are used to name these particular views. They set the task of studying the use of a model in specific contexts of application. A recent defense of such a deflationary view is put forward by Bas van Fraassen and his main contention is a so-called "Hauptsatz" of representation: "There is no representation except in the sense that some things are used, made, or taken, to represent some things as thus or so" (van Fraassen 2008, 32). Although some see a conflict between the two perspectives on representation they can be regarded as complementary rather than as excluding each other (cf. Chakravartty 2010). One reason is that only if there is a certain informational connection between target and vehicle then the preferred use of one model over another in order to learn about the target could be justified. In order to answer questions of why one model is a better representational vehicle than another one an informational standpoint is needed. And on the other hand, the question of whether a certain model is a good representation as such is ambiguous. A representational vehicle like, e.g., the harmonic oscillator can be used to model different kinds of phenomena for different purposes. Here, a standpoint, which incorporates the study of specific uses, is needed in order to individuate a specific representation that is achieved with the help of a model.

\section{Suárez's Inferential View and the Means of Representation}

Suárez endorses a so-called "inferential" account of scientific representation that is devoted to the practice of model building. He notices a "diverse range of models and modeling techniques employed in the sciences" (Suárez 2010, 92). In order to understand the activity of scientists it is vital for this account to study actual uses of models in specific contexts of application. Furthermore, the purposes of scientists that use and develop models are of highest relevance according to this position. Simultaneously the study of general properties of representational vehicles or general relations between these vehicles and their respective targets is shifted to concrete relations that scientists actually employ when modeling a phenomenon. The inferential conception is characterized by two conditions for a vehicle to represent a target. These conditions are meant to provide only "surface features" instead of universal necessary and sufficient conditions that are present in every instance of scientific representation (cf. Suárez 2004, 771). These two features are (a) the representational force, and (b) the capacity of the vehicles to allow inferences about the respective target systems, the inferential capacity. The first condition is grounded in the scientists' practice of using representational tools like models or other vehicles. In the course of this practice scientists establish an asymmetry in the relation between models and targets. Beyond that, the norms of the respective community ensure that there is a restricted determination of targets of these representational models. Suárez calls the asymmetry in the relation the "essential directionality" of representation (Suárez 2004, 767). This directionality assures that the force consists in the models pointing to the targets and not vice versa (cf. Suárez 2010, 98). The second condition of inferential capacity should assure that via studying representational models scientists are able to learn something about the respective target systems. Vehicles should allow inferences about these target systems. Suárez agrees with Chris Swoyer that successful models should allow surrogative reasoning (cf. Swoyer 1991). Besides, this phenomenon of representational vehicles, to allow inferences about their targets, is a feature stressed by other accounts of representation, that 
could be subsumed either under the label of informational or under a label of a use account (cf. Contessa 2007; Hughes 1997).

A related value of representational vehicles that Suárez stresses is the cognitive value of "objectivity." According to Suárez, scientific representations are cognitively valuable because they "provide us with specific information regarding their targets" (Suárez 2004, 772). The representations possess this property of objectivity, which means that they enable understanding of certain phenomena or targets. The objectivity of representation guarantees that one can learn something about the world via studying representational vehicles. Because of that feature, scientific representation cannot be established through mere stipulation as, for example is possible in cases of linguistic representations (cf. Suárez 2003, 227). Beyond that, representational vehicles must possess an internal structure and their parts and relations must be interpretable in terms of the target's parts and relations. ${ }^{4}$ Furthermore, there has to be a standard for correctness of inferences from the vehicles to the targets that is grounded in the practice of a certain scientific community (cf. Suárez 2010, 98).

Suárez makes a distinction between means and constituents of representation and this distinction underlies at least four of his arguments against similarity views of representation. He is looking for an account of the means of representation whereas he portrays some or maybe all of his opponents as trying to explicate the constituents of representation. This distinction is unfortunately not well explicated. Very roughly the difference seems to be one between a constituent as a general relation and a means as a specific relation. Looking for a more precise characterization of the contrast leaves one with puzzling hints only. Suárez gives the following definitions of these two kinds of relations.

At any time, the relation $\mathrm{R}$ between $\mathrm{A}$ and $\mathrm{B}$ is the means of the representation of $\mathrm{B}$ by $\mathrm{A}$ if and only if, at that time, $\mathrm{R}$ is actively considered in an inquiry into the properties of $\mathrm{B}$ by reasoning about $\mathrm{A}$. [...] The relation $\mathrm{R}$ between $\mathrm{A}$ and $\mathrm{B}$ is the constituents of the representation of B by A if and only if R's obtaining is necessary and sufficient for A to represent B. (Suárez 2003, 230)

The means may be interpreted as a concrete token of a representational relation, because Suárez characterizes it as the relation between a vehicle $A$ and a $\operatorname{target} B$ that is actively considered in an inquiry. The constituent as a general relation between vehicle and target may be interpreted as a relation type in contrast to a token. Or perhaps the constituent may be seen as a universal in contrast to a means as a particular. It is not clear whether the first contrast, between type and token, the second one, between universal and particular, or even a further contrast is aimed at. There is another quote that makes it even more difficult to understand what Suárez means by "means":

[T]here may be a great variety of means by which representation does its work: isomorphism and similarity are just two common ones, but there are others, such as exemplification, instantiation, convention, truth. (Suárez 2003, 229)

Here, Suárez lists examples for other means. As Suárez defines means as relations between vehicles and targets this raises the question in which ways instantiation and convention are relations between these two relata. Furthermore, he points out that "an object $A[\ldots]$ may hold more than one type of relation to another $B$, but at any one time only one of these will

\footnotetext{
4 This feature is also stressed by Contessa. In his interpretational account of representation an interpretation of a model in terms of a target is the defining constituent of the notion of epistemic representation (cf. Contessa 2007).
} 
be the means of representation" (Suárez 2003, 229). From this follows that even if one examines a single pair of model and corresponding target it is possible that different scientists may actively consider different relations between these two relata. Therefore one model can yield various means of representation depending on the scientists using that model. Even a single scientist is able to consider different relations after each other. These relations are then means that must be taken with an index of time and in order to differentiate them from other scientists' means there must be a second index for users as well.

Suárez uses the notion of means in his first argument against informational views of scientific representation. The Argument from Variety should reveal that there are many different means of representation that cannot be accounted for by similarity or structural views of representation. Suárez uses different examples of apparently successful scientific representations in order to show that the means of representation are not captured by a similarity or a structural view in each case. For example, he claims that the case of a scale model of a bridge shows the inadequacy of structural views. Here, the means is a similarity relation and therefore a structural view cannot account for this means.

It is by reasoning on the basis of these similarities that the source [the vehicle, in my terminology] does its representational work. [...] By contrast isomorphism, which is well-defined only as a relation between mathematical structures, does not apply directly to the relation between two physical objects described in case 1 [The case of scale model and bridge]. But it does apply to some abstract structures that are exemplified by these two objects, such as their geometric shape. [...] The means of the relation of representation are not in this case captured by the [structural] conception because this conception misidentifies its relata, which are the physical objects themselves, and not the structures exemplified. (Suárez 2003, 231)

In contrast to the scale model, another case, a graph of a bridge, can be accounted for by the structural view because here isomorphism is the means of representation.

[A] piece of paper containing the graph of a bridge is only similar to the bridge it represents with respect to the geometric shape and proportions between the different points; nothing else is interestingly similar. This "similarity of structure" is better captured by the alternative [structural] conception [...]. Maps, plans and graphs are typical cases where isomorphism is the means of scientific representation. (Suárez 2003, 231)

So in one case a similarity view is the appropriate account and in the other case a structural view is the better account. None of the two is the better account for both examples. Beyond that, Suárez also gives two more examples, the Billiard Ball Model of a gas as a representation of a system of molecules and the representation of a quantum system by a quantum state diffusion equation. Also these cases purport to show that none of the accounts can explain all instances of representation.

Although similarity and isomorphism are among the most common means of representation in science neither one, on its own, covers even nearly the whole range. (Suárez 2003, 231)

The conclusion of the argument is that no single view is capable of explicating the variety of means of representation. "[I]t follows that neither [the structural] nor [the similarity view], on their own, can account for the means of scientific representation" (Suárez 2003, 229). There is the problem of interpreting the distinction between means and constituents 
and the missing proper understanding of means. It is difficult to evaluate Suárez's argument and its conclusion. Nevertheless, the following comments may be made. Why are the exemplified structures in the case of the scale model and bridge not possible relata of a similarity or isomorphism relation? At first sight, it seems that both views could say something meaningful about this case of representation. The second case seems to point in the same direction. Here, also similarity and structural views may both be applicable. Suárez himself diagnoses a "similarity" between points on paper and the geometric shape of the bridge. Because the similarity is a structural similarity and it is only limited to a geometrical shape the structural view is assessed as "better" as the similarity view. However, this is not reason enough to exclude the similarity view.

Consider again the first case of the scale model of a bridge. If one takes a means as a relation that is relative to a user then it could be the case that one user $X$ really considers a structural relation between a structure that is exemplified by the scale model and a structure that is exemplified by the bridge. Another user $Y$ might consider a property that the model and the bridge share, say the ratio of the height to its length. Does this really show that there are different relations that are used in the activity of representation in case of user $X$ and in case of user $Y$ ? If the question is answered in the affirmative then we need at least two theories of means of representation, namely a theory of the means of user $X$ and a theory of the means of user $Y$. If there are different moments in time to take into account and other user dependent means, as well, then the number of theories might be multiplied even more. This reasoning shows that such an understanding of means leads to the difficulty of finding a single or even a small number of theories of the means of representation. There is no reason to show that a similarity view and a structural view of representation are not capable of explaining both these successful representations of a bridge with the help of various vehicles. Beyond that, if one asks what unites these phenomena then a similarity view might provide a good answer. According to a sophisticated similarity view these two means of user $X$ and $Y$ could be seen both as forms of similarity. The best explanation of scale model and bridge is then that it is a case of representation because model and bridge stand in the relation of similarity to each other. Prima facie, the Argument from Variety is not convincing.

\section{Substantive Reductionist Views and Arguments Against Sufficiency}

\subsection{Reductionist and Other Similarity Views}

The major group of opponents that Suárez faces is constituted by adherents of reductionist views on representation. These views focus on the relation of representation and they "aim to radically naturalize the notion of representation" (Suárez 2003, 225). To naturalize a concept in this context amounts to reducing it to facts. In order to do that, the reductionist has to show that the concept does not depend upon purposes or value judgments of agents. Furthermore, Suárez characterizes the reductionist views as "substantive." A substantive view tries to formulate a single necessary and sufficient condition of scientific representation. The substantive view poses a constitutional question regarding representation, namely: what is the relation between a vehicle and a target that constitutes representation? (cf. Suárez 2010, 92).

Suárez strongly argues against the explication of representation with the help of "similarity" or related notions. He attacks a position that can be regarded as an ideal type 
of a similarity view. According to this position a vehicle $A$ represents a $\operatorname{target} B$ if and only if $A$ is similar to $B$. In fact, he faces two opposing positions, the position using the aforementioned slogan and another one that uses "isomorphism" instead of "similarity." He labels these positions as Sim and Iso, respectively, and they read as follows:

Sim: A vehicle represents a target iff the vehicle is similar to the target.

Iso: A vehicle represents a target iff the structure exemplified by the vehicle is isomorphic to the structure exemplified by the target. (cf. Suárez 2003, 227)

As I reconstruct Suárez, he delivers six arguments against these two positions of Sim and Iso: the Logical Argument (1), the Argument from Mistargeting (2), the Non-Sufficiency Argument (3), the Argument from Quantitative Inexactness (4), the Non-necessity Argument (5) and the Argument from Variety (6). I divide the arguments into three groups. One group is constituted by arguments directed at similarity as a sufficient condition for representation. The second group encompasses arguments against similarity as a necessary condition. And the third one consists of the Argument from Variety already discussed in the foregoing section. Suárez refers to the arguments as touchstones for a theory of representation. He claims for example that a "satisfactory theory of representation must defeat these [...] arguments" (Suárez 2004, 768). ${ }^{5}$ The arguments are in the first place meant to counter naturalistic reductions of representation. However, Suárez does not use the arguments for this purpose only. In the 2003 paper and in later papers, as well, he applies the arguments to other positions besides the reductionist approaches. For example, he tries to amend the two attacked positions of Sim and Iso and in the course of that amendment he discusses nonreductionist positions that he labels as Sim' and Iso' respectively. Proponents of these positions do not follow the aim of naturalizing representation (cf. Suárez 2003, 238). ${ }^{6}$

In the following, I will subsume the positions of Sim and Iso under the single heading of "similarity views," when possible. This is justified because isomorphism can be regarded as a special form of similarity. The reason for this classification is that the relation of similarity is reflexive and symmetric and the isomorphic relation between two structures is reflexive, symmetric and transitive. So, the isomorphic relations are a subclass of the class of similarity relations. Occasionally, Suárez himself concedes that. "It is possible in general to understand isomorphism as a form of similarity" (Suárez 2003, 228) and he even states that "isomorphism is a case of similarity" (Suárez 2003, 232). In other contexts, however, he stresses that similarity and isomorphism are different means that are exploited in particular instances of representation.

Suárez's reason for attacking the very thin looking positions of Sim and Iso seems to rest also on the distinction between means and constituents. According to Suárez, discovering the constituent of representation is the aim of a substantive account of representation. Thus,

\footnotetext{
5 In this quote and in the original paper he speaks of five arguments. I count them as six because I divide his originally called Argument from Misrepresentation into two, namely Argument from Mistargeting and Argument from Quantitative Inexactness. This is motivated by them aiming at different conditions of representation, at a sufficient and at a necessary condition respectively.

6 The positions of Sim' and Iso' involve an additional clause and by that deliver sufficient conditions of representation. This has the consequence that "the non-sufficiency argument no longer applies" and, beyond that Suárez ponders whether "the logical argument might also lose its force" (Suárez 2003, 238). He stresses that the other arguments are applicable to these non-naturalistic positions, nonetheless. "But the other arguments still apply. The non-necessity argument is, if anything, strengthened, as the necessary conditions on representation are now stronger. The argument from variety shows that neither [Iso'] nor [Sim'] can describe all the means of representation; while the misrepresentation and non-necessity arguments show that they do not provide a substantial theory of the constituents of representation" (ibid.).
} 
philosophers endorsing a substantive view are looking for a relation that is both necessary and sufficient for a vehicle to represent a target. So as to face the simplest forms of such a substantive view Suárez draws the picture of his opponents above that might be regarded as a naive view on representation. ${ }^{7}$

It appears that similarity views like Sim or Iso are not very widespread. Particularly the claim that similarity is sufficient for representation seems to be hardly convincing at first sight. ${ }^{8}$ And in this ideal form, these theses lack a single proponent. However, recently, Adam Toon (2012) reconstructed Ron Giere's similarity view of representation as providing a sufficient condition of that notion that has similarity as one conjunct. Beyond that, there are scholars who claim that certain formal interpretations of similarity are sufficient for representation. Andreas Bartels (2006) argues that a homomorphism is sufficient for a potential representation and Steven French (2003) questions the claim that isomorphism is not sufficient for representation.

Especially the requirement of similarity as a necessary condition for representation is intuitively compelling. In Sect. 5, the underlying question that will be asked is whether it is possible to establish an account of scientific representation that comprises similarity as a necessary condition in the sense of left-to-right reading of the biconditional of Sim. First, let us examine Suárez's arguments against similarity as a sufficient condition. There are three arguments against similarity as a sufficient condition for representation that Suárez gives. They are the Logical Argument (1), the Argument from Mistargeting (2) and the Non-Sufficiency Argument (3).

\subsection{Arguments Against Similarity as a Sufficient Condition}

(1) The Logical Argument. Suárez assumes that there is a general class of representations of which scientific representation is a subclass. Beyond that, Suárez speaks of "ordinary" representations but he does not explicitly state what the ordinary representations are. It seems that he regards primarily paintings as prototypical examples of ordinary representations and that scientific representations are a subclass of ordinary representations. Suárez claims that representational relations in general are not reflexive, not symmetric, and not transitive. Scientific representation therefore possesses these properties of not being reflexive, not being symmetric, and not being transitive.

A substantive theory must make clear that scientific representation is indeed a type of representation; i.e. that it shares the properties of ordinary representation. Representation in general is an essentially non-symmetric phenomenon. [...] Representation is also non-transitive and non-reflexive. (Suárez 2003, 232.)

Furthermore, he suggests that representation might be irreflexive, asymmetric, and intransitive. But he does not want to argue for that (cf. ibid.). Although Suárez distances himself from developing a substantive theory, he does not renounce the demand mentioned in the first sentence in the preceding quote. In line with this he uses examples from the fine arts to exemplify the logical properties of representation. Diego Velázquez's painting of Pope Innocent $\mathrm{X}$ is a representational vehicle that has the Pope as its target. The painting

\footnotetext{
7 This is the name Goodman (1968) gives to a closely related position concerning pictorial representation. In fact, he even dubs it the most naive view.

${ }^{8}$ Consider a pair of twins. If similarity were sufficient for representation then one twin would represent the other. This is an unwelcome consequence of similarity being sufficient for representation and this example can be used in a reductio argument against the claim of Sim (cf. Goodman 1968,4).
} 
represents the Pope. This representational relation is not reflexive because the painting does not represent itself. Neither is it symmetric because the Pope does not represent the painting. And finally another painting is used to indicate that this representation is not transitive. This is shown by Francis Bacon's variation on Velázquez's painting that represents the original painting but not the Pope. In using these examples Suárez is able to show that these paintings and maybe paintings in general have the requested properties of ordinary representations.

The argument can be summarized as follows: because Sim and Iso cannot account for the logical properties of ordinary representation, both views are inadequate. In fact, according to the two accounts, representation is at least reflexive and symmetric. And this in turn contradicts the claim that representation possesses the logical properties of not being reflexive and not being symmetric. This is at first sight a convincing argument and because of that it seems to be indeed effective against the right-to-left reading of the biconditional of Sim. Granted that works of art are paradigmatic representations and that scientific representations and these representations share the same logical properties it is compelling. The take home message of this argument is: similarity is not sufficient for representation.

Although I introduced similarity in the sense of a symmetric and reflexive relation there are other approaches that use it in a way that it is open for similarity to be not symmetric (e.g., Weisberg 2013). Empirical reasons for this decision are psychological studies of similarity judgments. A non-symmetric similarity relation that was empirically investigated is for example the estimated similarity between China and North-Korea (cf. Tversky 2004, Ch. 1, 3). Participants in psychological studies endorsed the claim that North-Korea is more similar to China than China is to North-Korea. If you think that this allows inferring the conclusion that China really is less similar to North-Korea than North-Korea is to China you may find the following argument interesting and not a purely sophistic afterthought.

To employ an example from a movie by Roman Polanski: the similarity relation between the baby of Rosemary, which she conceived by the devil, and the father of the baby could be assessed as not symmetric. A possible reductio argument goes as follows. If Rosemary 's baby is similar to its father and if similarity is symmetric then the father must be similar to his child. Yet, there is a strong intuition that a father is not as similar to his baby as the baby is similar to him and moreover there is an even stronger intuition that the devil is not similar to a baby. Hence, the reductio argument may lead to similarity being not symmetric. A possible reply could be to point out that this particular asymmetry is grounded in a further relation, maybe a causal one. Or another reply could be that literally similarity is symmetric but there are special uses of the notion that suggest an understanding of it with an asymmetry built into that relation (cf. van Fraassen 2008, 18).

(2) The Argument from Mistargeting. In this argument, Suárez treats a problem of misrepresentation that leads to considering the wrong target. He again illustrates the argument by invoking a situation using Velazquez's painting as a vehicle of representation. Suppose that a person looks roughly similar to Pope Innocent $\mathrm{X}$ as depicted in the painting. To think of the painting as a representation of that person is a mistake. Suárez calls it "mistargeting" and he concludes that the similarity view of representation cannot explain why this situation involves a misrepresentation. According to Sim, the painting should be regarded as a representation of the person because it is similar to him. The similarity view yields that this apparent misrepresentation is not a misrepresentation at all. And this looks like an absurd consequence of this view. In particular, what this example shows is that Sim cannot distinguish between accidental and other forms of similarity. The view is not able to 
assure that the vehicle refers to the target. Furthermore, like in the preceding argument, the stress is only on the right-to-left direction of the biconditional of Sim. There may be similarity between two relata without reference. This is the lesson that can be learned from the Argument from Mistargeting. The take home message is the same as the one from the Logical Argument: similarity is not sufficient for representation.

This reasoning presupposes that this particular misrepresentation is not a representation at all. And certainly common sense would agree. The painting is not a representation of the person. However, the question is what that means for scientific cases. In debates over scientific representation most scholars assume that misrepresentation is a form of representation. Here a difference pointed out by Andreas Bartels and Robert Cummins may help. According to these authors, successful representation involves representational content and reference that provides the respective target (cf. Bartels 2006; Cummins 1996). In this case, the mistake is that the vehicle does not refer to the target. In most scientific cases of misrepresentation, the vehicles refer to their targets while the content is not in order. ${ }^{9}$

(3) The Non-Sufficiency Argument. Actually the two other arguments already revealed that there are severe problems for a naive similarity view that aims at accounting for a sufficient condition of representation. In order to be thorough let us quickly go through these next points.

First, Suárez stresses that the two accounts have nothing to say about the representational force. The feature of essential directionality "lies at the heart of the phenomenological non-symmetry of the representational relation" (Suárez 2003, 236). Because neither Sim nor Iso can capture this directionality they do not provide sufficient conditions for representation. Neither Sim nor Iso can explain why the vehicle points to the target and not vice versa.

In a second line of reasoning especially Iso is under concern. Suárez assumes that it is solely possible for an object to exemplify a structure if the object stands in a particular relation to the structure. Now according to Iso, if two objects stand in a representational relation there has to be an isomorphic mapping from one structure to another structure. A second assumption is that the objects themselves cannot stand in this isomorphic relation to each other. Each object needs to exemplify a structure to do so. The relation between the object exemplifying a structure and the structure itself is a problem for Iso because this particular account only covers the relation between structures and it cannot explain this additional relation. Van Fraassen displays an analogous argument, which he calls the "Loss of Reality" objection (cf. van Fraassen 2008, 258).

What we need to ask ourselves now, though, is [...] how an abstract entity can represent something physical, so as to make sense of it in this concrete context. [...] How can we answer the question of how a theory or model relates to the phenomena by pointing to a relation between theoretical and data models, both of them abstract entities? The answer has to be that the data model[s] represent the phenomena. (van Fraassen 2008, 252f.)

Structural accounts want to explain the relation between on the one hand a theory or model and on the other hand a phenomenon or target with the help of certain mapping relations between structures. There may be an isomorphism between a theoretical model and a data

\footnotetext{
9 This kind of misrepresentation is used in the Argument from Quantitative Inexactness (see Sect. 5.1). Aside from that there are misrepresentations in science that are mistaken because of failures in reference. These cases like e.g. ether or phlogiston are lacking any referent at all.
} 
model if these models are understood as structures. The relation that one originally wants to cover is the relation between model and target. One wants to explain how the model represents the target. However, a structural account cannot claim that the data model represents the target because representation is a mapping relation between structures and the target is not a structure. So, according to the structural similarity view of representation the relation between data model and target cannot be called a representation relation.

Both arguments point to a specific problem for structural accounts like Iso. The relation between objects and structures is something that such formal accounts cannot cover. For example, the chain of isomorphisms between different models in a hierarchy of models does not reach to the target. The route from theoretical models to data models cannot bridge the gap to the target itself. One possible answer to that challenge is that the relation between data model and target does not have to be called a representation relation. Maybe it is enough to speak here of exemplification. The target exemplifies a structure and the data model is just such a structure that may be exemplified by the target. The theory or model represents the target via the structural relation between theoretical model and data model and the additional relation of exemplification between target and data model. A slight variant to that answer could be that the theoretical model is similar to the target by way of the morphism between theoretical model and data model and the exemplified structure of the target that is accounted for by the data model. ${ }^{10}$

In summary, most of the arguments seem to be correct conditional on generally accepted assumptions. The arguments may indeed show that similarity is not sufficient for representation. But, even if similarity would be not sufficient for scientific representation that did not rule out the relation to constitute a necessary condition for scientific representation. Let us turn now to the arguments regarding necessary condition.

\section{Arguments Against Similarity as a Necessary Condition}

Two of the six arguments are directed at similarity as a necessary condition for representation. These two are the Argument from Quantitative Inexactness and the Non-necessity Argument (See Sect. 5.2). Let's start with the Argument from Quantitative Inexactness

\subsection{The Argument from Quantitative Inexactness}

This argument touches on the problem of misrepresentation in modeling. Suárez acknowledges two forms of misrepresentation. One form is the phenomenon of mistargeting that was already discussed in the previous section. The other form is the phenomenon of inaccuracy, or "inexactness" as I want to call it.

The second form of misrepresentation is the even more ubiquitous, perhaps universal, phenomenon of inaccuracy. Most representations are to some degree inaccurate in some or other respects. [...][Sim] requires that the target and the source must share some although not necessarily all their properties. Hence [Sim] can account for the type of inaccuracy that arises in an incomplete or idealised representation of a phenomenon, i.e. one that leaves out particularly salient features such as the highly idealised representation of classical motion on a frictionless plane. But

${ }^{10}$ Thanks to an anonymous reviewer for pressing me on that issue. 
this will not always help to understand inaccurate representation in science, where the inaccuracy is much more often quantitative than qualitative. (Suárez 2003, 234f.)

In some cases of misrepresentation similarity accounts can explain why an inaccurate model is a representation of a target. For example, incomplete or idealized representations leave out certain properties that are not important. Yet, the models and the targets share important properties. And so the models and targets are similar to each other. Suárez calls this type of inaccuracy "qualitative." He concedes that similarity views can account for this qualitative inaccuracy. In contrast, quantitative inaccuracy poses a problem. In science, most cases of inaccuracies involve these quantitative inaccuracies. The conclusion of the argument is that similarity views cannot explain quantitative inaccuracies in scientific modeling. The example of Newtonian mechanics without relativistic corrections providing a representation of the solar system is a case in point. There is a variance between predictions and observations of planetary motions.

The interesting question is not what properties fail to obtain, but rather how far is the divergence between the predictions and the observations regarding the values of the properties that do obtain. [Sim] offers no guide on this issue. (Suárez 2003, 235)

The guidance that Suárez seems to be missing can be interpreted as a quest to degrees of similarity. Let's see whether a similarity view can give an answer to that.

In the following discussion of the argument I am going to use a distinction that was first put forward by Paul Teller (2008) in order to make sense of the fact that modeling involves idealizations and abstractions and, still, can lead to representations that are accurate enough depending on certain purposes. Teller distinguishes precise or imprecise claims from accurate or inaccurate claims (cf. Teller 2008, 436). He furthermore uses a more general predicate, namely the term "inexact." Claims that are inexact may either be imprecise or inaccurate (or both). With this distinction, he tries to justify the use of certain inexact claims in order to state something that may be imprecise but still accurate enough. Teller takes ordinary language claims about the height of people as an example. If one claims that a person's height is six feet then this claim could be interpreted as meaning either that the height is six feet precisely or that it is six feet close enough. Given that no human being is precisely six feet tall, this claim is unsuccessful in the way that it is either false because no one is precisely six feet tall or that in a certain context a more accurate information is needed than just six feet close enough. So, the claim may be false because it is imprecise or false because it is inaccurate (cf. Teller 2008, 438). However, in another context this second vague interpretation of the claim may be accurate enough although it is not precise. So, interpreted as six feet close enough and used in a context where one only wants to distinguish between, say heights around five feet and heights around six feet, the claim may be imprecise yet accurate enough.

Let us now apply these notions to the Newtonian modeling. A Newtonian model allows inferring certain descriptions of planetary motions. These descriptions that Suárez calls "inaccurate" are in the terminology introduced above first of all inexact descriptions. Yet, although these descriptions are inexact still the models built from the Newtonian theory represent the planetary motions accurately enough for certain purposes. Suárez himself concedes that Newtonian mechanics can "provide an approximately correct representation of the solar system" (Suárez 2003, 235). So, while this modeling does not allow a precise description of the planetary motions, it nevertheless can lead to a description that is accurate enough, at least for certain purposes. 
Precisely because similarity is a notion that admits to degrees it is well suited to cover this case of inexactness. The characterization of sharing a property could be used to talk about this divergence between prediction and observation. Given that some value $X$ is the calculated value of the prediction one could add an error term $\Delta$. This leads to a quantitative property $X \pm \Delta$. With the help of this further variable margins of error around the value $X$ can be defined. Ronald Giere notices the use of these margins of error as one strategy to deal with the problem that strictly speaking mathematical equations are often false when they are used as descriptions of empirical facts.

The margins of error rarely appear in the descriptions or calculations until one gets to the point of comparing theoretical predictions with actual measurements. When it comes time to compare the abstract model with reality, the deltas may then be understood as specifying the degree of similarity [...] between the abstract model and the real system. (Giere 1999, 50)

These margins of error can then be seen as established degrees of similarity in specific contexts like, e.g., the context of evaluating measurement outcomes. Of course, it is not written into Sim directly how small this error term should be. The definite interval cannot be fixed beforehand. What counts as accurate enough depends on concrete cases. Notice that this is a move that reductionist accounts might not support. This move seems to involve at least some implicit or explicit value judgments that determine what is accurate enough.

A reductionist may try to give a stricter definition of the different forms of inexactness. Let us go back to Teller's example of height and try to sharpen the notion of accuracy. I propose to shift the scale of feet to the scale of meters and to reason with decimals. Suppose, we are again talking about the height of a person, we may say that she is $1.80 \mathrm{~m}$ tall. First of all this is probably inexact because the person's exact height is most likely different from $1.80 \mathrm{~m}$. The number of the height will have more non-zero digits. Say our person is precisely $1.81523 \mathrm{~m}$ tall. In that case our claim would be not that precise but could still be accurate enough depending on how many correct digits the claim contains. For example in a context in which the first two digits are required to be correct the above claim is accurate enough. If we require three correct digits and we follow the standard rule of rounding then we should better say that she is $1.82 \mathrm{~m}$ tall. In that context of the three required correct digits our above sentence is false. The person is not 1.80 but $1.82 \mathrm{~m}$ tall. Yet, one could claim that it is less inaccurate than say that she is $1.90 \mathrm{~m}$ tall. Can a reductionist be satisfied with this solution? Still, there is the notion of required number of correct digits and this requirement has to be based on a normative claim. A view that does not allow an explication of representation that involves value judgments cannot tolerate such a move. So, the hard reductionist has to find another answer to the argument.

A non-reductionist similarity view, in contrast, can answer the challenge of the quantitative inexactness. In fact the apparent weakness of similarity views turns out to be a virtue. Although some models like the Newtonian models mentioned above might be inexact representations, they still do not have to be regarded as inaccurate. These representations can be seen as accurate enough. It is a virtue of similarity that it can be used to make sense of the claim that Newtonian mechanics can represent planetary motions accurately enough. Although the theory delivers no descriptions that are perfectly precise it nevertheless represents the planetary motions because its models are similar to the phenomenon. This case can be made efficient for the non-reductionist opponent's point of view, contrary to Suárez. Thus, in order to state that similarity is a necessary condition for scientific representation there need to be established degrees of similarity. Margins of error 
that are used, for example, to evaluate measurement outcomes can be regarded as established degrees in scientific practice. Similarity may be not sufficient for representation. Yet, it may still be tenable to defend the claim that similarity is necessary, given specific degrees of similarity.

\subsection{The Non-necessity Argument}

Given that similarity is simply constituted by two things sharing any property it is trite to state that everything is similar to everything. This is the starting point of the Non-Necessity Argument.

It is trivial that any object is in principle similar to any other object. In fact the point is often made that if all logically possible properties are permitted, then any object is similar to any other object in an infinite number of ways, i.e. there is an infinite number of properties that we can concoct that will be shared between the objects. [...] If so, similarity would be necessary for representation but in a completely trivial way. For it would not only be a necessary condition on representation but also on non-representation. (Suárez 2003, 235)

If any object is similar to any object similarity is not only a necessary condition for representation but also for non-representation, this is indeed a trivial result. In response to that, Suárez assesses a possible reply for an advocate of a similarity view. This reply consists in stressing the aspect of relevance. Only the relevant similarities are matters of concern.

A restriction is needed here to only those properties or aspects of the source and the target that are "relevant" to the representational relation: A represents B if and only if A and B are similar in the relevant respects. It is not the case that any source is in principle trivially similar in the relevant aspects to what it represents. (ibid.)

In fact, the similarity theorist only needs to claim that the relevant similarity is a necessary condition and not a necessary and sufficient one. So, the claim that she can endorse is: a vehicle represents a target only if vehicle and target are similar in the relevant respects. However Suárez does not want to stop here. He presses the similarity theorist on the notion of relevance. A further attack against the similarity account is to ask what the criterion of relevance actually is. Suárez assumes that this criterion must establish a link between relevance and the representational relation and he imagines a possible explication of that link. His "improved" similarity slogan is: "A represents B if and only if A and B are similar in those respects in which A represents B" (Suárez 2003, 235). This is obviously circular. If the similarity theorist subscribed to this view she might be in trouble.

Yet, a similarity theorist does not have to agree to this slogan. She only needs to endorse the above claim about the necessary condition of relevant similarity. The crucial question at this point is whether the endorser of a similarity view must provide a general criterion of relevance. Here, a specific stance to relevance that acknowledges the practice of science might be enough. The relevant respect in which two things such as models and targets are similar to each other is a contextual issue. Research questions define relevant properties that are studied in scientific practice. The relevant respects are given before modeling starts and because of that no further analysis of the notion is required.

Suárez comes to a parallel conclusion but again he besets the similarity theorist once more. He presents an example from the fine arts in order to show that relevant similarity is not necessary for representation. 
The defender of similarity might retort that 'relevance' is a fully intuitive notion of straightforward application in practice; a primitive notion in no need of further analysis. That this is not so is made most vivid in the analogy with art, and to illustrate this point I like to invoke Guernica, the well-known painting by Picasso. [...] The point is that none of the targets of Guernica can be easily placed in the relevant similarity relation with the painting, and mutatis mutandis for isomorphism.

(Suárez 2003, 235f.)

A second point in the argument is the relation between mathematical equations and the targets the equations describe. According to Suárez, this is not a relation of similarity. "An equation-i.e., the actual physical signs on the paper-is as dissimilar as it could be from the phenomenon that it represents" (Suárez 2003, 236). So, there are two counterexamples against the claim of relevant similarity as a necessary condition of scientific representation. The first is a work of art and the second is a mathematical equation. Both do represent corresponding targets but they are not similar to their targets.

In order to evaluate this argument, a short notice is in order. The first example is not a representation in the sciences. It may be that it is a counterexample to a broad similarity view of representation. My short reply to it is that if you are interested in scientific representation then this is not a legitimate example. Linguistic entities do also represent but hardly anyone would claim that a term is similar to what it refers to, e.g., the term "cats" is clearly not similar to certain animals (cf. Chakravartty 2010, 200). ${ }^{11}$ The second counterexample may be a representation in the sciences. If the equation is used in order to represent a specific phenomenon in the context of a modeling task then it is a representational vehicle. Besides, it may indeed also be regarded as a linguistic entity. ${ }^{12}$ Granted, as a syntactical unit an equation is not similar to a target. However, to claim that it is even seems to be a category mistake. No defender of similarity would claim that this is the required relation in modeling. Yet, an equation is not simply regarded as a syntactic entity. In the wake of the semantic view of theories a shift from uninterpreted calculi to mathematical structures is made. The objects of study of philosophers of science following the semantic view are not primarily linguistic entities. If an equation is used in a scientific context then this equation specifies a mathematical structure. In this context, the structure can be regarded as the model of the target system. And such a structure might indeed be similar to the target if the equation under scrutiny is used to adequately represent the phenomena. Maybe there is even an isomorphic mapping from the structure to a data model of the target. Yet, other mappings could be more adequate. Someone who endorses a similarity account is not committed to Iso or related views that declare isomorphism to be a necessary condition for representation.

This argument does not show that similarity cannot be conceptualized as a necessary condition of scientific representation, either. Especially if relevant respects are conceded, the similarity theorist is not in trouble.

\footnotetext{
11 Chakravartty discusses the case of linguistic representation and the example of the term "cats" and the corresponding animals. He ponders over the interpretation of this representation as involving a form of similarity, too. Specifically, he reflects about the relation between the semantic content of linguistic expressions and corresponding targets in the world.

12 The question whether mathematical representation counts as linguistic or not is usually answered in the affirmative by scholars following the syntactic view of scientific theories. Followers of the semantic view take the other direction. A recent handbook entry on representation in science follows this second route (Teller 2008).
} 


\section{Conclusion and Discussion}

Suárez's rejection of an explication of scientific representation with the help of similarity seems to be based on his belief that, in order to explicate the notion this way, similarity has either to be the means or the constituent of representation. If similarity is the constituent then it must be necessary and sufficient for representation. The first three arguments are used to show that similarity is not sufficient. According to these arguments, similarity cannot be the constituent of representation. The Argument from Variety is used to show that similarity is not in all cases of representation a means of representation. According to that argument, similarity is not the means of representation. I have argued that the Argument from Variety is not decisive for the question whether similarity is or is not the means of representation.

Suárez's arguments against similarity as a necessary condition for scientific representation could be satisfactorily answered especially from a non-reductionist point of view. So, similarity may be conceptualized as a necessary condition for scientific representation granted that relevant respects and degrees of similarity are conceded. While similarity may not be necessary and sufficient for representation it might be defended that it is necessary, still. Similarity may not be the constituent of representation but nevertheless it is a relation that may be used to explicate scientific representation. The arguments did not show the following thesis false: only if a vehicle is similar to a target in relevant respects and to a certain degree of similarity then the vehicle is a scientific representation of that target.

The question as to whether there are other necessary conditions for a vehicle to represent a target remains open. Likewise, the study whether there are several necessary conditions that are jointly sufficient is a task for further research.

As a prospect of a positive account of similarity being a necessary condition I would like to end with mentioning how the considered condition can be compared to other proposed conditions. Besides similarity scholars discuss other conditions for representation in the sciences like interpretation (Hughes 1997; Contessa 2007) or inferential capacity (Contessa 2007; Suárez 2004). If one compares the different proposed conditions for scientific representation similarity, inferential capacity and interpretation then similarity looks as the best option to take because it can give a ground for the other conditions. Especially, similarity is more fundamental than the condition of inferential capacity. Similarity can be used in order to explain why representational models could have an inferential capacity in the first place. In a nutshell, only because there is a similarity between vehicle and target, it is justified to derive certain surrogative inferences from the vehicle to the target. Similarity between models and targets is a reason for models to have the capacity to lead to correct inferences about the targets. Without the similarity relation, the inferences from models to target systems can hardly be justified. It may be true that an interpretation of a model in terms of a target can also explain why a model can lead to inferences about the target. Yet, that is not sufficient to ground the correctness of the inferences. It would be a miracle to reach correct claims about a target with the help of a model if the model were not similar to the target.

Acknowledgments I would like to thank Daniel Baron, Raoul Bußmann, Florian Fischer, Rafaela Hillerbrand, David Koepsell, Peter Kroes, Stephan Padel, Marianne Richter and two anonymous reviewers for helpful comments and suggestions on earlier drafts of the paper.

Open Access This article is distributed under the terms of the Creative Commons Attribution 4.0 International License (http://creativecommons.org/licenses/by/4.0/), which permits unrestricted use, distribution, 
and reproduction in any medium, provided you give appropriate credit to the original author(s) and the source, provide a link to the Creative Commons license, and indicate if changes were made.

\section{References}

Bartels, A. (2006). Defending the structural concept of representation. Theoria, 55, 7-19.

Bueno, O., \& French, S. (2011). How theories represent. British Journal for the Philosophy of Science, 62, 857-894.

Chakravartty, A. (2010). Informational versus functional theories of scientific representation. Synthese, 172, 197-213.

Contessa, G. (2007). Scientific representation, interpretation, and surrogative reasoning. Philosophy of Science, 74, 48-68.

Cummins, R. (1996). Representations, targets, and attitudes. Cambridge, MA: MIT Press.

French, S. (2003). A model-theoretic account of representation (Or, I Don't Know Much about Art... But I Know It Involves Isomorphism). Philosophy of Science, 70, 1472-1483.

Giere, R. N. (1999). Using models to represent reality. In L. Magnani \& N. J. Nersessian (Eds.), Modelbased reasoning in scientific discovery (pp. 41-57). New York: Kluwer.

Goodman, N. (1968). Languages of art. An approach to a theory of symbols. Indianapolis: Hackett.

Hughes, R. I. G. (1997). Models and representation. Philosophy of Science, 64, 325-336.

Suárez, M. (2003). Scientific representation: Against similarity and isomorphism. International Studies in the Philosophy of Science, 17, 225-244.

Suárez, M. (2004). An inferential conception of scientific representation. Philosophy of Science, 71, $767-779$.

Suárez, M. (2010). Scientific representation. Philosophy Compass, 5, 91-101.

Swoyer, C. (1991). Structural representation and surrogative reasoning. Synthese, 87, 449-508.

Teller, P. (2008). Representation in science. In S. Psillos \& M. Curd (Eds.), The routledge companion to philosophy of science (pp. 435-441). New York: Routledge.

Toon, A. (2012). Similarity and scientific representation. International Studies in the Philosophy of Science, $26,241-257$.

Tversky, A. (2004). In E. Shafir (Ed.), Preference, belief, and similarity: Selected writings. Cambridge, MA: MIT Press.

van Fraassen, B. C. (2008). Scientific representation: Paradoxes of perspective. Oxford: Oxford University Press.

Weisberg, M. (2013). Simulation and similarity: Using models to understand the world. New York: Oxford University Press. 